\title{
Cardiac Rehabilitation and Survival for Ischemic Heart Disease
}

\author{
Rebecca Lolley ${ }^{1,2}$ (1) . Daniel E. Forman ${ }^{1,3,4,5}$
}

Accepted: 21 September 2021 / Published online: 6 November 2021

(c) The Author(s), under exclusive licence to Springer Science+Business Media, LLC, part of Springer Nature 2021

\begin{abstract}
Purpose of Review Cardiac rehabilitation (CR) referral is a Class I post-myocardial infarction (MI) recommendation from the American Heart Association and the American College of Cardiology, yet referral rates remain strikingly low, with cardiologists some of the worst under-referring offenders. This paper seeks to review the evolution of CR and its well-established benefits, as well as reasons behind the poor referral and utilization.

Recent Findings CR is a secondary prevention program for cardiovascular disease (CVD) that was first initiated in the 1970s as a hospital-based exercise program after an acute MI, but then evolved into a comprehensive multi-disciplinary program for patients with a wider range of cardiovascular diseases. CR mortality and morbidity benefits have endured over decades, even as interventional and pharmacological cardiovascular therapeutics have improved and as patients have become relatively more stable.

Summary Despite being an evidence-based clinical standard, referral and participation in CR are disconcertingly low. In efforts to combat poor referral rates, and improve care in the contemporary care environment, the approach to CR is evolving. Innovations include broadening CR beyond the hospital setting into remote- and hybrid-based formats, while still incorporating exercise training, risk factor reduction, and education, as well as behavioral and psychosocial support. Nonetheless, there still remain many challenges to overcome in order to increase participation of all ages, financials, races, and sexes. With new performance measures as well as an increasing number of NIH-funded studies on the horizon, there is hope that $\mathrm{CR}$ will become a relatively more valued and utilized component of cardiovascular preventative care.
\end{abstract}

Keywords Cardiac rehabilitation (CR) $\cdot$ Stable ischemic heart disease (IHD) $\cdot$ Myocardial infarction (MI) $\cdot$ Cardiovascular disease (CVD)

This article is part of the Topical Collection on Ischemic Heart Disease

Rebecca Lolley

rebecca.lolley@bannerhealth.com

Daniel E. Forman

formand@pitt.edu

1 Division of Cardiology, University of Pittsburgh Medical Center, Pittsburgh, PA, USA

2 Cardiovascular Institute of North Colorado, Banner Health, 1800 15th St Suite 310, Greeley, CO 80631, USA

3 Department of Medicine, University of Pittsburgh, Pittsburgh, PA, USA

4 Department of Medicine, VA Pittsburgh Healthcare System, Pittsburgh, PA, USA

5 Section of Geriatric Cardiology, University of Pittsburgh Medical Center, 3471 Fifth Avenue, Suite 500, Pittsburgh, PA 15213, USA

\section{Introduction}

Contemporary cardiac rehabilitation (CR) is a comprehensive multi-disciplinary program to reduce morbidity and mortality in cardiovascular patients. Key benefits also include reduced rehospitalization and improvements in quality of life and function. CR referral is a Class I recommendation from the American Heart Association and the American College of Cardiology for patients which initially included only those with recent myocardial infarction (MI) and post-coronary artery bypass grafting (CABG), which has expanded over time to also include post-percutaneous coronary intervention (PCI), chronic stable angina, valvular heart disease, heart transplant, heart failure with reduced EF, and, most recently, PAD patients. Although recommendations for CR now extend to a wide range of patients, ischemic heart disease (IHD) remains one of the most common indications for the referral, and CR participation continues to demonstrate significant benefits, beyond those achieved with even 
the latest advances in revascularization and adjunctive medical therapies. Notably, CR itself has also changed over time as it responded to research showing the complex physiology underlying cardiovascular disease (CVD); CR expanded from what was originally only exercise training to now also including education, behavior modification, risk factor reduction, stress relief, and other vital therapeutic goals. However, despite the enduring and even growing benefits of CR for IHD, referral rates and participation remain low, particularly due to under referral of many eligible patients. This review evaluates the evolution of CR and its benefits, particularly in respect to patients with IHD.

\section{Evolution of Cardiac Rehabilitation}

When formalized CR began in the 1970s, it revolutionized care for the acute myocardial infarction (MI) patient. In the early 1900s, therapeutic options were sparse and cardiac morbidity and mortality were exceptionally high. Survivors typically had sustained fully completed infarcts, with high prevalence of residual ischemia, large territories of myocardial scar, and arrhythmic vulnerabilities. Given these risks, treatment norms usually entailed 6 weeks of bed rest and long hospital stays. However, over time it came to be recognized that these long periods of inactivity resulted in significant deconditioning, pulmonary emboli, depression, and progressive clinical deterioration. In the 1940s, chair therapy was first introduced enforcing that myocardial infarction patients simply sat up in a chair instead of laying recumbent in bed [1]. This progressed to allowing short daily walks 4 weeks after acute coronary events throughout the 1950s. In 1968, Saltin et al. published the Dallas Bed Rest and Exercise Study which showed that bed rest reduced $\mathrm{VO} 2 \mathrm{max}$ through reductions in stroke volume and cardiac output, while exercise training enhanced $\mathrm{VO} 2 \mathrm{max}$ by increasing cardiac output [2]. These and other studies contributed to the evolution of exercise recommendations post-MI [3, 4]. With many patients and physicians still fearful about initiating exercise in a non-supervised fashion, the concept for formal CR was formally conceptualized in 1970. Participation in a supervised CR setting provided a process to both promote and guide functional recovery for patients who in most cases were still intrinsically unstable.

In its early stages during the 1970s and 1980s, the objectives of CR were primarily to improve physical activity and help patients with IHD reengage with their lives. Many were deconditioned and anxious after cardiac events that had left them feeling debilitated and vulnerable. CR was geared particularly towards the middle-aged males, with the goal to get them back to work. Related CR literature focused exclusively on males [5]. In the early 1980, 1990s, and 2000s, many studies were starting to show benefits and possibly mortality benefit for exercise-based CR [1, 6]. In 2000, Cochrane published a systematic review of studies ranging from the 1980s-1999 and concluded that exercise-based $\mathrm{CR}$ is effective in reducing cardiac deaths [7]. However, the authors noted that the evidence was weakened by the poor quality of trials and specifically no randomized control trials. Trails also continued to enroll predominantly white, middleaged males who were at lower risk for complications than the general public. Furthermore, many wondered if more could be done to enhance $\mathrm{CR}$ effectiveness.

Amidst these prevailing considerations, it became increasingly appreciated that CVD patients were burdened not only by acute events but by underlying conditions that increased CVD risks. Hypertension and diabetes were among the factors that were associated with CVD that could also potentially be addressed as part of CR. The first Clinical Practice Guidelines for Cardiac Rehabilitation expanded the scope of CR to include strategies to reduce modifiable CVD risk factors [8]. Soon after, the INTERHEART study proved that specific aggressive risk factor modification can reduce the risk of MI, reinforcing rationale for broader CR programmatic goals [9]. The authors concluded there were nine prominent modifiable risk factors (dyslipidemia, smoking, hypertension, diabetes, abdominal obesity, psychosocial factors, consumption of fruits and vegetables, alcohol use, and regular physical activity) which account for over $90 \%$ of the risk for MI [4]. This study helped pave the way for the current core components of risk factor modification into $\mathrm{CR}$. Tobacco cessation and nutritional and weight loss counseling in combination with cholesterol, diabetes, and blood pressure management are all mainstays within contemporary CR programs and were officially added to the American Association of Cardiovascular and Pulmonary Rehabilitation's performance measures in 2007 [10]. With the incorporation of risk factor modification to exercise therapy, $\mathrm{CR}$ has evolved to become a comprehensive lifestyle cardiovascular risk reduction program.

\section{CR Benefits}

\section{A. Mortality Benefit}

While advancing therapeutics remains one of cardiology's great success stories, the fact that CR has endured as an enhancement of care beyond all the other components of conventional therapy is remarkable. Over the decades since CR was initially formulated, there have been numerous advancements in treatment of IHD, both technically and pharmacologically, all improving outcomes. Mortality-associated CVD has significantly decreased [11] and bed rest as well as hospitalization length of stay has decreased as well, moderating debilitation that was once notorious. Acute MIs are 
recognized and treated earlier with revascularization, antiplatelets, beta-blockers, and statins. PCI has become progressively more technically advanced with improvement in stent durability, pliability, and deployment. CABG surgeries are more streamlined with less time on bypass, yielding faster recoveries and hospital stays. Nevertheless, among those who continue on to $\mathrm{CR}$, outcomes are still significantly improved compared to those who do not attend CR.

In the most recent 2018 Cochrane systematic review on "Exercise-based cardiac rehabilitation for coronary artery disease," 63 different randomized control trials (RTC) were evaluated to assess the effectiveness of exercise-based CR compared with usual care on mortality, morbidity, and quality of life in CVD. The authors found that cardiovascular mortality and hospitalizations were still both significantly reduced in patients who underwent exercise-based CR when compared to controls [12•]. Health-related quality of life was also significantly improved in the CR groups. Of note, the trials included in the Cochrane Reviews have almost exclusively been exercise-only programs, and benefits from multifaceted CR programs are presumed by many to be likely even more significant. These findings echoed assessments from the previous Cochrane reviews published in 2001 [7] and 2011 [13] which also demonstrated significant mortality benefits and decreased hospitalizations for the exercise CR groups. Notably, total mortality was still not significantly improved. Again, such accounts are muddled by the fact that many of the trials studied pertained only to exercise-only CR programs and did not account for the added benefits which may be attributable to multifaceted CR programs. Furthermore, the populations studied were predominantly lower risk men following revascularization, which may underestimate potential all-cause mortality benefits of CR in other clinical contexts and populations. Methodologic weaknesses were inherent to the CROS-II study published in 2020. This study evaluated CR trials through 2018, and demonstrated $\mathrm{CV}$ mortality-lowering benefits. The authors also conclude that the studies varied significantly in their outcome measurements and highlighted need to determine internationally accepted standards for CR [14].

\section{B. Secondary Risk Factor Reduction}

The Cochrane and CROS-II reviews have emphasized CR mortality-lowering benefits of $\mathrm{CR}$, but other benefits increasingly recognize additional benefits. Declines in cholesterol level and blood pressure and smoking reduction [15] as a result of CR are also reported. These reductions can occur in programs that include exclusively exercise, but are putatively enhanced by programs that include education, smoking cession, diet, and behavioral modifications as part of the CR in a multi-disciplinary approach. Multi-disciplinary programs tend to emphasize nutritional assessments throughout the stages of a $\mathrm{CR}$ program, assessing baseline caloric intake as well as dietary content, allowing for key individualized dietary modifications and goals to be established. With obesity on the rise across the globe and recognized as an independent factor for CVD, importance of weight management is now often emphasized. Cholesterol and diabetes education also tend to be completed throughout CR as well. Monitoring these risk factors allows for healthcare providers to tailor medications for optimal benefit and in combination with CR care has been found to improve outcomes of these measures [16]. Tobacco cessation is also addressed in multi-disciplinary $\mathrm{CR}$, with assessments involving the readiness to quit, as well as intervention with education and counseling.

\section{Functional Improvements}

Regaining one's functional independence post-MI has been an important goal since the initiation of CR. Increase in patient functional status was a primary critical observation and now included as a secondary and even primary endpoint in many RCT. This has been extremely important as CR has expanded to include patients with heart failure and peripheral arterial disease, and also as more patients with CVD are older and/or frail. One of the first studies to formally assess functional status was published in 2003 utilizing the well-validated Medical Outcomes Study Short Form-36 (SF-36) evaluating 700 patients after 6 months $[17,18]$. The authors found that CR after was associated with significant improvements in physical functioning as well as adoption of secondary prevention measures. $\mathrm{CR}$ was also found to improve long-term maintenance of healthy lifestyle including participating in regular exercise and modifying diet, allowing patient to successfully return to their prior lifestyles, sometimes even healthier [18]. An especially frail and vulnerable population post hospital discharge are the older adult heart failure patients. Cardiac rehabilitation can be especially important for these patients as they transition to home. A recently published study, REHAB-HF, showed that heart failure patients (both with reduced and preserved ejection fraction) hospitalized for acute decompensated heart failure benefited greatly with an early, tailored, and individualized CR intervention. Patients with early CR showed improvements in Short Physical Performance Battery scores as well as decreases in hospitalizations [19]. Of note however, this study was an exercise-only intervention with one-on-one physical therapy. Additional research is needed evaluating the expanded multidomain forms of CR accessible today, and would likely show even greater benefit. 


\section{Medication Management}

There have been numerous pharmacologic contributions to the secondary prevention and reduction in cardiovascular mortality over the last several decades with the addition of aspirin, P2Y12 inhibitors, beta-blockers, and cholesterolreducing medications. Statins alone have been attributed to reduce mortality by $25-40 \%$ after 6 months of use, however adherence rates are less than $50 \%$ at 1 year after initiation [20]. The addition of risk assessments and counseling components to $\mathrm{CR}$ programs has allowed for an important educational period for patients on adherence of their CVD medications, leading to important reductions in blood pressure, glucose, and cholesterol [16].

\section{E. Psychosocial Impact}

Stress and depression have been identified as substantial risks for future cardiovascular events, with up to $30 \%$ of the population's attributable risk of MI linked to stress [9]. Depression and anxiety also contribute to a significant amount of morbidity post-MI, occurring in up to $20 \%$ of patients [21]. Fortunately, exercise has been shown to significantly reduce stress and depression, as well as improve quality of life and emotional well-being, and has therefore been assessed throughout the existence of CR. In contemporary CR programs, stress management workshops and tailored social interventions are also commonly offered to improve patient outcomes [22].

\section{CR Continued Challenges/Improving Poor Utilization}

\section{A. Shifting Strategies in the CR Process}

Early CR was first organized and developed in the inpatient setting, but with improvements in overall cardiac care and decreased admission times, expansion beyond the hospital became an apparent and a growing necessity. In response to these needs, CR advanced into a three-phase operation in the 1990s, with phase I still focusing on inpatient care with early mobilization of the patient and encouraging risk factor modification and follow-up. Phase II evolved as a supervised ambulatory outpatient program consisting of 12 weeks of formalized CR with persistent risk factor reduction as mentioned previously. Phase III is conveying the importance and giving the patient instructions on how to maintain life-long health and exercise [23]. Despite the rationale for this process, it has had some ironic detrimental effects over time. Phase I of CR largely faded away or was eliminated at many centers due to shorter length of stay of hospitalizations. The loss of phase I also had impact on phase II, as the whole notion of coming back to the hospital for phase II CR often seems unnecessary to patients and physicians after state-ofthe-art PCIs and medications have created an impression of cure. Studies on CR participation have shown that there is a large lack in systematic referral, especially given competing demands with family priorities, high costs from copays, and transportation challenges as additional impediments [24].

New delivery strategies of CR have been used to try and overcome these barriers. The initiation of home- and other remote- or home-based formats for CR can sometimes transcend barriers of distance and scheduling conflicts and has opened new implementation advantages. A recent study in the VA population showed that when given the opportunity to choose home-based or a center-based approach, almost $50 \%$ preferred the home-based method [25]. Nonetheless, even with more flexible approaches to CR, enrollment has not significantly increased. Many patients and providers are less comfortable with remote-based options, especially due to concerns about safety, lack of provider contact, and lack of social interaction with other patients [26]. Limitations in patient monitoring, and even in the care that is delivered in remote-based programs, are also key concerns. However, remote-based CR is rapidly evolving, especially in since COVID-19 limited the use of on-site programs [27, 28], with the hope that safety, connectedness, and quality of care can all be optimized with advancing technology and experience [29].

\section{B. Difference in Referral}

Despite the well-defined and persistent benefit that CR has on cardiovascular mortality and quality of life, it remains widely underutilized. Reasons for poor utilization reflect multiple layers of barriers between the healthcare systems, patients, and clinician and are likely multifaceted and complex. Notably, rates of underutilization and referral are not equal across specialties and procedure performed. This has especially been the case in post-PCI referral in comparison to post-CABG and valvular surgery referral. In a recent outcome assessment of hospital level variation in CR referral, patients post-CABG were referred $91 \%$ of the time in comparison to only $48 \%$ of post-PCI patients [30]. Investigators speculated this is due to the higher perceived need for CR after surgery as part of surgical recovery, in comparison to low perceived need for CR by cardiologists for PCI procedures. In other words, $\mathrm{CR}$ was prescribed mostly to catalyze recovery from the procedural burden of surgery, but not for fundamental CVD. PCIs are often outpatient procedures or only one-night hospitalizations, with the tacit messaging from providers to patients that the procedure is so welltolerated that there is no need for CR [9]. Nonetheless, the post-CABG population clearly demonstrates that consistent and persistent emphasis of $\mathrm{CR}$ is possible and associated 
with high patient participation, suggesting that cardiologists could like due much to improve CR referral after PCI.

\section{Referral Inequalities}

In addition to post-procedural differences in referral, there is a large discrepancy in the referral to CR for women, racial disparities, and older adults. As mentioned previously, most of the early studies done on CR almost exclusively include middle-aged lower risk men post-MI. In 2014, a meta-analysis showed that women are $36 \%$ less likely to enroll in CR when compared to men [31]. Racial disparities are especially prevalent with Blacks, Hispanic, and Asian patients respectively 20\%, 36\%, and 50\% less likely to receive referral than whites [32]. Arguably one of the highest risk groups for recurrent $\mathrm{CV}$ events is the older adult population, but their participation in CR has been found to be less than $13 \%$ nationally [33]. In order to address this problem, many studies are now looking at new ways to increase enrollment into CR programs. In a recent Cochrane review of these studies published in 2019, enrollment was most successful if delivered by face-toface encounters and done by healthcare or allied healthcare professions (physiotherapists) [34••]. To help implement these findings, an online version has even been developed to assist healthcare professionals with discussing CR at

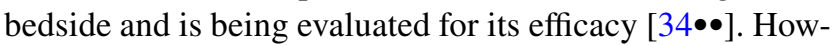
ever, to use these tools, there need to be more urgency and awareness in hospitals, healthcare providers, and entire health systems to uphold their responsibility of enrolling patients and relaying the importance of $\mathrm{CR}$ in their future health.

Barriers related to access have substantially affected enrollment and utilization, including those without transportation, living in remote areas, and lacking financial resources and childcare [35]. Even if patients get enrolled, sustainability of engagement due to these barriers has proven to be significantly restrictive with inflexibility of schedules or lack of insurance [36]. Although remote options of CR have been offered as a solution and are increasingly available, constraints of access to computers and internet or even the use of technology still create significant barriers.

\section{CR Engagement}

Enrollment is not the only problem facing CR participation for patients; maintaining patient engagement in programs to receive the full benefits is another challenge. Of the less than $20 \%$ referred, less than $20 \%$ of those patients complete all 36 prescribed sessions. Whereas reports suggest that patients who complete more sessions of CR have better outcomes in a dose-related fashion [37, 38], i.e., allowing more time to build exercise capacity and other wellness benefits. However, these studies may also reflect a dimension of selection bias, as those who attended the full 36 sessions may have other relevant characteristics that affect outcomes than solely differences in the CR session participation. Overall, there needs to be more research evaluating the barriers to why patients are not completing fully prescribed sessions, as well as more emphasis from health systems on not only enrollment but maintenance.

\section{Conclusions and Future Directions}

Just as overall CVD care has been revolutionized over the past 100 years, CR has come a long way since it was initially conceptualized in the early 1900s. From chair therapy to now a focus on comprehensive lifestyle transformation, $\mathrm{CR}$ is an extremely important part of secondary prevention in patients with CVD. There are still many challenges to overcome, including overcoming the perceived lesser importance of its effects among medical and technological advances, and increasing enrollment of all ages, financial stability, races, and genders, as well as reinforcing the importance attending the full treatment regimens. There is also a great need for standardization of measuring outcomes and performance across CR studies. A set of comprehensive Quality Indicators (QIs) was recently developed in China where $\mathrm{CR}$ is also underutilized and is promising to better standardize research [39•]. In the USA, CR has now been added to HEDIS quality performance measures and may even lead to financial enticement to encourage participation. There has also been a proliferation of NIH grants in CR in the last few years in order to address the difference in exercise regiments (high vs. low intensity programs), behavior (of providers and patients), and disparities of care (gender, race, and age), as well as technologies (wearables, apps, and other aspects of virtual care). These are all oriented towards improving efficacy and implementation, as well as standardization of CR. Overall, the investment in these grants demonstrates that there is an implicit affirmation and explicit enhancement of CR as an important part of IHD care.

Acknowledgements Dr. Forman receives funds from the National Institute of Aging through grants R01AG060499, R01AG058883, U19AG065188, and P30AG024827.

\section{Compliance with Ethical Standards}

Conflict of Interest The authors declare no competing interests. 
Human and Animal Rights and Informed Consent This article does not contain any studies with human or animal subjects performed by any of the authors.

\section{References}

Papers of particular interest, published recently, have been highlighted as:

- Of importance

$\bullet$ Of major importance

1. Levine SA, Lown B. "Armchair" treatment of acute coronary thrombosis. JAMA. 1952;148(16):1365-9.

2. Saltin B, Blomqvist G, Mitchell JH, et al. Response to exercise after bed rest and after training. Circulation. 1968;38:VII1-78.

3. Bethell HJ. Cardiac rehabilitation: from Hellerstein to the millennium. Int J Clin Pract. 2000;54:92-7.

4. Naughton J, Lategola MT, Shanbour K. A physical rehabilitation program for cardiac patients: a progress report. Am J Med Sci. 1966;252:545-53.

5. Wannamethee SG, Shaper AG, Walker M. Physical activity and mortality in older men with diagnosed coronary heart disease. Circulation. 2000;102:1358-63.

6. Naughton J. Exercise training for patients with coronary artery disease. Cardiac Rehabilitation revisit. Sports Med. 1992;14:304-19.

7. Jolliffe JA, Rees K, Taylor RS, Thompson D, Oldridge N, Ebrahim S. Exercise-based rehabilitation for coronary heart disease. Cochrane Database Syst Rev. 2000;(4)

8. Wenger NK, Froehlicher ES, Smith LK, et al. Cardiac rehabilitation: clinical practice guidelines. Rockville, MD: Agency for Health Care Policy and Research and the National Heart, Lung, and Blood Institute; 1995.

9. Yusuf S, et al. Effect of potentially modifiable risk factors associated with myocardial infarction in 52 countries (the INTERHEART study). Lancet. 2004;364:937-52.

10. Thomas RJ, King M, Lui K, Oldridge N, Piña IL, Spertus J, ACC/AHA Task Force Members. AACVPR/ACC/AHA 2007 performance measures on cardiac rehabilitation for referral to and delivery of cardiac rehabilitation/secondary prevention services. J Cardiopulm Rehabil Prev. 2007;27(5):260-90.

11. Puymirat E, Simon T, Cayla G, Cottin Y, Elbaz M, Coste P, et al. Acute myocardial infarction: changes in patient characteristics, management, and 6-month outcomes over a period of 20 years in the FAST-MI Program 1995 to 2015. Circulation. 2017;136(20):1908-19.

12. Anderson L, Thompson DR, Oldridge N, Zwisler AD, Rees K, Marin N, et al. Exercise-based cardiac rehabilitation for coronary heart disease. Cochrane Database of Systemic Reviews. 2018. Findings from this updated review exemplify the continued benefits of CR for ischemic heart disease and specifically showed cardiovascular mortality and hospitalizations were both significantly reduced in patients who underwent exercise-based CR.

13. Heran BS, Chen JM, Ebrahim S, Moxham T, Oldrige N, Rees $\mathrm{K}$, et al. Exercise-based cardiac rehabilitation for coronary heart disease. Cochrane Database Syst Rev. 2011;(7).

14. Salzwedel A, Jensen K, Rauch B, et al. Effectiveness of comprehensive cardiac rehabilitation in coronary artery disease patients treated according to contemporary evidence based medicine: update of the Cardiac Rehabilitation Outcome Study (CROSII). Eur J Prev Cardiol. 2020;27(16):1756-74. https://doi.org/ 10.1177/2047487320905719.

15. Taylor RS, Brown A, Ebrahim S, et al. Exercise-based rehabilitation for patients with coronary heart disease: systemic review and meta-analysis of randomized controlled trials. Am J Med. 2004;116:682-92.

16. Balady GJ, Williams MA, Ades PA, Bittner V, Comoss P, Foody JM, et al. American Heart Association Exercise, Cardiac Rehabilitation, and Prevention Committee, the Council on Clinical Cardiology; American Heart Association Council on Cardiovascular Nursing; American Heart Association Council on Epidemiology and Prevention; American Heart Association Council on Nutrition, Physical Activity, and Metabolism; American Association of Cardiovascular and Pulmonary Rehabilitation. Core components of cardiac rehabilitation/secondary prevention programs: 2007 update: a scientific statement from the American Heart Association Exercise, Cardiac Rehabilitation, and Prevention Committee, the Council on Clinical Cardiology; the Councils on Cardiovascular Nursing, Epidemiology and Prevention, and Nutrition, Physical Activity, and Metabolism; and the American Association of Cardiovascular and Pulmonary Rehabilitation. Circulation. 2007;115(20):2675-82.

17. Ware JE, Sherbourne CD. The MOS 36-item short-form health survey (SF-36). Med Care. 1992;30:473-83.

18. Pasquali SK, Alexander KP, Coombs LP, Lytle BL, Peterson ED. Effect of cardiac rehabilitation on functional outcomes after coronary revascularization. Am Heart J. 2003;145:445-51.

19. Kitzman DW, Whellan DJ, Duncan P, Pastva AM, Mentz RJ, Reeves GR, et al. Physical rehabilitation for older patients hospitalized for heart failure. N Engl J Med. 2021;385(3):203-216. https://doi.org/10.1056/NEJMoa2026141. Epub 2021 May 16. PMID: 33999544; PMCID: PMC8353658.

20. Hirsh BJ, Smilowitz NR, Rosenson RS, Fuster V, Sperling LS. Utilization of and adherence to guideline-recommended lipidlowering therapy after acute coronary syndrome: opportunities for improvement. J Am Coll Cardiol. 2015;66(2):184-92.

21. Milani RV, Lavie CJ, Cassidy MM. Effects of cardiac rehabilitation and exercise training programs on depression in patients after major coronary events. Am Heart J. 1996;132:726-32.

22. Denollet J, Brutsaert DL. Enhancing emotional well-being by comprehensive rehabilitation in patients with coronary heart disease. Eur Heart J. 1995;16:1070-8.

23. Squires RW, Gau GT, Miller TD, Allison TG, Lavie CJ. Cardiovascular rehabilitation: status, 1990. Mayo Clin Proc. 1990;65(5):731-55.

24. Ruano-Ravina A, Pena-Gil C, Abu-Assi E, et al. Participation and adherence to cardiac rehabilitation programs. A systematic review. Int J Cardiol. 2016;223:436-43.

25. Tang LH, Berg SK, Christensen J, Lawaetz J, Doherty P, Taylor RS, Langberg H, Zwisler AD. Patients' preference for exercise setting and its influence on the health benefits gained from exercise-based cardiac rehabilitation. Int J Cardiol. 2017;232:33-9.

26. Rohrbach G, Schopfer DW, Krishnamurthi N, et al. The design and implementation of a home-based cardiac rehabilitation program. Fed Pract. 2017;34(5):34-9.

27. Van Iterson EH, Laffin LJ, Crawford M, Mc Mahan D, Cho L, Khot U. Cardiac rehabilitation is essential in the COVID-19 era: delivering uninterrupted heart care based on the Cleveland clinic experience. J Cardiopulm Rehabil Prev. 2021;41(2):88-92.

28. Thamman R, Janardhanan R. Cardiac rehabilitation using telemedicine: the need for tele cardiac rehabilitation. Rev Cardiovasc Med. 2020;21(4):497-500. https://doi.org/10.31083/j.rcm. 2020.04.201. PMID: 33387993.

29. Forman DE, LaFond K, Panch T, Allsup K, Manning K, Sattelmair J. Utility and efficacy of a smartphone application to enhance the 
learning and behavior goals of traditional cardiac rehabilitation: a feasibility study. J Cardiopulm Rehabil Prev. 2014;34(5):327-34.

30. Beatty AL, Bradley SM, Maynard C, McCabe JM. Referral to cardiac rehabilitation after percutaneous coronary intervention, coronary artery bypass surgery, and valve surgery. Circ Cardiovasc Qual Outcomes. 2017;10.

31. Samayoa L, Grace SL, Gravely S, Scott LB, Marzolini S, Colella TJ. Sex differences in cardiac rehabilitation enrollment: a metaanalysis. Can J Cardiol. 2014;30(7):793-800. https://doi.org/10. 1016/j.cjca.2013.11.007. Epub 2013 Nov 12 PMID: 24726052.

32. Li S, Fonarow GC, Mukamal K, Xu H, Matsouaka RA, Devore $\mathrm{AD}$, Bhatt DL. Sex and racial disparities in cardiac rehabilitation referral at hospital discharge and gaps in long-term mortality. J Am Heart Assoc. 2018;7:e008088. https://doi.org/10.1161/ JAHA.117.008088.

33. Schopfer DW, Forman DE. Cardiac rehabilitation in older adults. Can J Cardiol. 2016;32(9):1088-96.

34.• Pio CSA, Chaves G, Davies P, Taylor R, Grace S. Interventions to promote patient utilization of cardiac rehabilitation: Cochrane systematic review and meta-analysis. J Clin Med. 2019;8(2):189. https://doi.org/10.3390/jcm8020189. Findings from this study indicate that interventions to increase enrollment in and adherence to CR can be effective, specifically when delivered face-to-face by healthcare providers.
35. Ragupathi L, Stribling J, Yakunina Y, Fuster V, McLaughlin MA, Vedanthan R. Availability, use, and barriers to cardiac rehabilitation in LMIC. Glob Heart. 2017;12:323-34.

36. Peters AE, Keeley EC. Trends and predictors of participation in cardiac rehabilitation following acute myocardial infarction: data from the behavioral risk factor surveillance system. J Am Heart Assoc. 2017;7(1):e007664.

37. Suaya JA, Stason WB, Ades PA, Normand SL, Shepard DS. Cardiac rehabilitation and survival in older coronary patients. J Am Coll Cardiol. 2009;54:25-33.

38. Hammill BG, Curtis LH, Schulman KA, Whellan DJ. Relationship between cardiac rehabilitation and long-term risks of death and myocardial infarction among elderly Medicare beneficiaries. Circulation. 2010;121(1):63-70.

39. Z Zheng X, Zhang M, Zheng Y, et al. Quality indicators for cardiac rehabilitation after myocardial infarction in China: a consensus panel and practice test. BMJ Open. 2020;10(12):e039757. https://doi.org/10.1136/bmjopen-2020-039757. Findings from this study indicate the effectiveness of quality indicators for CR as a way to improve and standardize utilization of CR.

Publisher's Note Springer Nature remains neutral with regard to jurisdictional claims in published maps and institutional affiliations. 